\title{
Histoire de la SHF
}

\author{
par Michel Banal, Max Perrin et Claude Thirriot
}

Un siècle d'histoire pour la Houille Blanche, presque autant pour la SHF (depuis 1912 !), une mission de reporter qui s'annonçait passionnante quand on nous l'a proposée puis arrivés au pied du mur, nous sommes effrayés par l'ampleur et la difficulté de la tâche. Pour bien faire ce serait l'affaire d'une thèse en Histoire des Sciences et Techniques (et pourquoi pas proposer ce thème à nos collègues historiens ?). La recherche des éléments bibliographiques est un vrai travail de bénédictin qui a pour échelle de temps l'éternité. Même dans le Saint des Saints de la SHF, les documents sur l'origine sont rares et épars. Aussi le survol de la vie de la SHF qui va suivre n'a pas la prétention ni même l'ambition de l'exhaustivité. Il veut être tout simplement un hommage à cette vieille dame (toujours jeune) qu'est la Société Hydrotechnique de France.

Elle est née en 1912 après une gestation de dix ans dans les pages de la Houille Blanche dirons-nous avec un peu d'imagination rétrospective. Sur son histoire, un document de synthèse en quelques pages a été écrit en 1990 par Michel Banal qui était déjà Président d'Honneur et plutôt que de plagier hypocritement ce texte, nous le citerons copieusement. Un autre texte non daté et non signé, à notre disposition, rapporte un épisode de l'histoire de la SHF toute jeune qui concerne l'étude des réseaux de distribution et du transport de l'électricité pendant la première guerre mondiale. Enfin une note presque effacée, d'une page et demie, qui porte en surcharge manuscrite la date 15 mai 1946, parle de la mutation de la SHF au moment de la création d'Electricité de France. Après 1946, les documents «biographiques" sont plus aisés à consulter. Il y a en particulier les longues litanies de toutes les réunions savantes et techniques organisées par la SHF appelées Sessions du Comité Technique et Journées de l'Hydraulique dont la lecture montre à la fois le foisonnement des thèmes d'intérêt et l'évolution des préoccupations nationales concernant l'eau.

En 1902, le premier Congrès de la Houille Blanche rassembla à Grenoble toutes les personnalités concernées par la création et l'exploitation des aménagements hydroélectriques : Directeurs de sociétés, ingénieurs, fonctionnaires, universitaires, etc. Tous les problèmes administratifs, financiers et techniques posés par ces aménagements furent examinés.

Une des suites données aux résolutions prises en conclusion du congrès fut la création d'une Commission des turbines rassemblant scientifiques, exploitants et constructeurs pour étudier les divers problèmes relatifs au rendement de ces machines et aux accidents survenus à certaines installations. Les travaux de cette commission atteignirent rapidement une telle ampleur que G. Cordier, Président de la chambre Syndicale des Forces Hydrauliques, fut incité à créer une société indépendante capable de posséder des fonds et de les gérer.

Telle est l'origine de la Société Hydrotechnique de France (SHF) créée le 22 mai 1912 sous le régime de la loi de 1901.

Dès le premier jour, la Société Hydrotechnique de France a eu la structure qu'elle devait conserver sans modification par la suite : en premier lieu, un ensemble d'adhérents permettant d'alimenter par leurs cotisations un budget géré par le Conseil d'Administration et, à côté, un Comité Technique composé des techniciens les plus qualifiés savants et ingénieurs recrutés par cooptation dans 1 'industrie, les laboratoires, les administrations et les Facultés, constituant l'organisme de travail de la Société, procédant aux études scientifiques ou techniques, prenant connaissance des communications qui lui sont soumises et organisant leur discussion. Le premier président du Comité Technique fut Henri Bazin, Membre de l'Institut, le savant bien connu.

Jusqu'à la nationalisation de l'énergie électrique, la Société Hydrotechnique de France a constitué l'organisme technique de la Chambre Syndicale des Forces Hydrauliques, qui réunissait toutes les entreprises ayant pour objet l'installation et l'exploitation des chutes d'eau pour la production de l'énergie électrique. Elle se présentait comme une société mi-savante, mi-technique. Ses statuts orientaient très nettement ses préoccupations vers l'hydroélectricité, elle devait, comme l'indique l'article 1 de ses statuts initiaux : «étudier toutes les questions, procéder à toutes recherches et expériences relatives à. l'hydraulique en vue, notamment, de permettre l'aménagement et l'exploitation des chutes d'eau dans les conditions les meilleures, les plus sûres et les plus économiques".

Parmi les problèmes à traiter en priorité, la communication $\mathrm{n}^{\circ}$ I en date de juillet 1913 du Conseil d'administration cite : la mesure des gros débits, l'évaluation des pertes de charge, les coups de bélier, le rendement des moteurs, les appareils spéciaux inventés pour renforcer les chutes, amorcer ou désamorcer les conduites, enregistrer les charges etc.

Dès son début, l'histoire de la SHF s'est donc trouvée liée très étroitement au «développement de l'industrie française des forces hydrauliques ».

Pendant la première guerre mondiale, la SHF fut sollicitée par le gouvernement pour contribuer à la substitution de l'énergie hydroélectrique au charbon dont l'approvisionnement devenait critique, et ce tout particulièrement à partir de 1916 dans le plus d'industries possibles et bien entendu dans celles de l'armement.

Citons quelques chiffres pour signifier la gravité de la crise énergétique alors rencontrée.

Avant 1914, la France consommait 60 millions de tonnes de charbon par an dont 40 millions de tonnes de production 
HISTOIRE DE LA SHF

Sessions du Comité Technique



nationale et 20 millions de tonnes d'importation. Les $40 \mathrm{MT}$ nationales, dont les $2 / 3$ provenaient des mines du Nord et de l'Est de la France, se trouvèrent réduites à 7 millions de tonnes par l'occupation ennemie et les dégâts causés aux mines par la guerre. L'importation en provenance de la seule Angleterre ne pouvait être accrue. En dépit des efforts pour développer la production des mines restées disponibles, un déficit important subsista dont furent victimes les particuliers et les industries non prioritaires. Le gouvernement mit en place en effet une organisation pour réserver le charbon disponible en priorité aux usines travaillant pour la défense nationale y compris à partir de 1917 les usines de production d'électricité.

A côté du charbon défaillant, l'Hydroélectricité apparaissait une ressource presque miraculeuse et les sociétés d'électrochimie et d'électrométallurgie virent leurs usines très sollicitées : conversion de leurs fabrications du temps de paix en production d'explosifs ou d'armement et transfert de leur production d'électricité sur des poudreries ou des usines d'armement créées par l'Etat. Lorsque la production des usines hydroélectriques devint insuffisante leurs exploitants furent incités à achever les usines dont les chantiers avaient été interrompus par la guerre puis à ouvrir de nouveaux chantiers. Pour aller plus loin dans l'économie de charbon on chercha à substituer l'électricité au charbon dans diverses productions. Enfin, on se préoccupa d'assurer une meilleure utilisation de l'énergie hydroélectrique, soumise à l'irrégularité aléatoire des précipitations et impossible à stocker (sauf en utilisant des grands réservoirs tout à fait exceptionnels à l'époque), en reliant les réseaux entre eux de manière à économiser le charbon pendant les périodes de bonne hydraulicité. Mais l'interconnexion des réseaux se heurtait à l'époque à des difficultés techniques et commerciales. Les difficultés commerciales provenaient des conflits d'intérêt entre les sociétés concessionnaires habituées plus souvent à se faire concurrence pour étendre leurs zones d'action qu'à coopérer et qui camouflaient souvent l'absence de volonté de coopération sous des difficultés techniques. Or ces difficultés techniques étaient réelles, la première étant la diversité des types de courant utilisés. Un bon exemple de la situation d'alors de la France. est donné par Paris où coexistaient les courants continu et alternatif triphasé et diphasé et ces derniers à 25 ,
42, $.50, .53$ périodes sans parler des tensions (en 1917 on relevait 60 tensions différentes utilisées en France entre 1000 et 60000 volts).

La première chose à faire était évidemment de remédier à cette situation en uniformisant autant que faire se pouvait les types de courant utilisés. Les responsables les plus lucides en étaient conscients depuis longtemps même si les intérêts de leurs sociétés ne les incitaient pas à prendre des initiatives à ce sujet: la guerre déclencha le mouvement. Dès la fin de 1916 quatre organismes professionnels mirent à l'étude l'unification des types de courant utilisés en France: Union des syndicats de l'électricité, le Syndicat des producteurs et distributeurs de l'énergie électrique, le Comité électrotechnique français et la Chambre syndicale des forces hydrauliques par l'intermédiaire de son organisme d'étude: la Société Hydrotechnique de France (SHF).

Ainsi de 1916 à 1921, la SHF eut un rôle fédérateur dans l'uniformisation des réseaux électriques. Dès le 16 juin 1917 , la SHF écrivit au Ministère des Travaux Publics pour recommander l'emploi du courant alternatif triphasé 50 périodes. Le Président de la sous-commission du Comité de l'électricité invita à son tour la SHF à poursuivre son étude en l'étendant au choix des tensions

La SHF participa aussi à l'établissement d'une carte des réseaux de distribution de l'électricité et elle paraît avoir été particulièrement fière de ce travail qui fut présenté le 14 juin 1920 à l'Académie des Sciences par M. Rateau, membre éminent de la SHF et constructeur de pompes fameux

Dans son rôle d'organisme technique, à la disposition d'une branche d'industrie, la SHF eut à étudier les problèmes pratiques auxquels se heurtaient tous les jours les constructeurs de matériel hydraulique et les exploitants d'usines hydroélectriques. De manière à faciliter leurs rapports et à, éviter d'inutiles contestations, elle établit successivement plusieurs cahiers des charges : pour la fourniture des turbines hydrauliques, pour la fourniture des conduites forcées en métal, ou en ciment armé, pour la fourniture des groupes électrogènes à turbines hydrauliques et des groupes élévatoires. Elle eut également à rédiger un «Code d'essais des installations hydrauliques " en vue d'unifier les modes d'application des différentes méthodes de mesures hydrauliques. 
Dans le domaine du service industriel, la SHF s'adjoignit, en 1927, un service d'essais et de contrôle permanent des installations hydrauliques. Il devait permettre aux entreprises de faire procéder aux essais de réception du matériel neuf et de faire vérifier, ensuite, les appareils en cours de service, de manière à déceler les diminutions de rendement qui pouvaient résulter de l'usure et du vieillissement.

Dans le domaine des études théoriques, en présence d'un ensemble de techniques qui n'en étaient encore qu'à leurs débuts, tous les problèmes se posaient à la fois : mesure des gros débits, évaluation des pertes de charge, études et détermination du rendement des turbines, étude de leur régulation, conditions de construction et d'établissement des conduites forcées, génie civil et bien d'autres questions. C'est au sein de la SHF que se développèrent notamment les premières grandes études sur les coups de bélier, les cheminées d'équilibre, les crues, les débits solides, limite de puissance des grosses machines hydrauliques.

En vue de renforcer son action dans le domaine des recherches, la Société Hydrotechnique de France fut amenée à créer un laboratoire, à Beauvert aux portes de Grenoble, dont l'inauguration eut lieu le 16 novembre 1922. Elle y fit procéder à des études sur les machines hydrauliques, sur l'écoulement dans les canaux, sur les pertes de charge, sur les mesures de débit. Le laboratoire comprenait une station de tarage des moulinets hydrométriques. La Société eut également à sa disposition le laboratoire du Saulcy, près de Metz.

Enfin, sous l'impulsion de P. Masse et de R. Gibrat, la SHF fut amenée à créer, en 1941, un Service d'Etudes Générales de Statistique et d'Hydrologie. Celui-ci eut à accomplir un important travail de documentation hydrologique : profils en long des cours d'eau, données relatives aux débits, corrélation et prévision des débits, et à procéder à diverses études d'économie hydroélectrique : modèle hydraulique, indices de valeur d'une chute d'eau, exploitation optimum des réservoirs. Comblant une lacune qui existait depuis 1920 , date où avait cessé la publication périodique assurée par le Ministère de l'Agriculture, la Société a réalisé l'édition régulière de l' " Annuaire Hydrologique de la France" dont le premier volume a donné les résultats de l'année 1939 et qui permet d'avoir, pour chaque année, une vue d'ensemble de l'hydraulicité de la France. Cette publication fut poursuivie jusqu'à l'édition en 1969 par les soins de l'administration d'un annuaire national des cours d'eau.

La nationalisation de l'énergie électrique influa sensiblement sur les destinées de la Société Hydrotechnique de France. Elle lui fit perdre son caractère semi-industriel et accentua son caractère de société savante, en limitant son activité à des études techniques et scientifiques.

Les services à objet industriel, tel le Service d'essais et de contrôle permanent des installations hydrauliques, ont été transférés à Electricité de France. Les Laboratoires du Saulcy et de Beauvert ont été fermés au profit du Laboratoire National d'Hydraulique installé par Electricité de France à Chatou, en collaboration avec la Direction des Voies Navigables et des Ports Maritimes conformément à un projet étudié par la SHF dès l'année 1942.

Les nouveaux statuts de la Société ont reflété sa nouvelle orientation, en la dégageant de toute préoccupation d'ordre industriel et en lui donnant, désormais, pour objet « L'étude de toutes questions relatives à l'hydraulique, à la mécanique des fluides et aux sciences qui s'y rattachent ».
Plus que jamais, l'essentiel de son activité s'exerce par les études de son Comité Technique. Constitué modestement à l'origine avec une vingtaine de membres, il en réunit, aujourd'hui, plus de 600 : hommes de laboratoires, hommes de chantiers, constructeurs de turbines et de conduites, entrepreneurs, fonctionnaires, jeunes ingénieurs côtoyant les plus éminents de leurs aînés. Le Comité Technique comporte plusieurs sections et commissions, qui se consacrent à des problèmes particuliers auxquels il est ainsi possible de donner une plus vive impulsion.

Dans le but d'encourager et récompenser ceux qui travaillent autour d'elle, la SHF a créé trois Prix actuellement annuels : le Prix Henri Milon (prix annuel d'Hydrologie), le Grand Prix d'Hydrotechnique et le Prix Valembois d'Hydromécanique

Outre les ouvrages édités directement pour la Société, les travaux du Comité Technique ont été et sont publiés dans les revues suivantes :

— de l'origine à 1936: Revue Générale de l'Electricité

— de 1936 à : 1939 : Revue Générale de 1'Hydraulique

— depuis 1948 : Revue La Houille Blanche

Ces revues diffusées dans le monde entier en particulier dans les bibliothèques des Services culturels des Ambassades de France ont favorisé le rayonnement de la technique et de la science française dans le domaine de l'Hydraulique et ont été l'amorce de relations personnelles entre les hydrauliciens étrangers et les auteurs français.

Pendant la seconde moitié du $\mathrm{XX}^{\mathrm{e}}$ siècle, la SHF a eu une activité débordante. Sa mission d'activité expérimentale au service des entreprises françaises avait pratiquement disparu en même temps que le laboratoire de Beauvert relayé par le démarrage du Laboratoire National d'Hydraulique de Chatou. Il resta cependant une activité coopérative qui était l'étude du rendement des machines hydrauliques animé par le groupe du même nom au sein de la SHF.

A partir de 1946 donc, les missions de la SHF furent de favoriser l'échange scientifique et technique entre les membres du Comité Technique et la diffusion des connaissances par l'organisation de réunions à la bonne périodicité. Ces réunions étaient essentiellement de deux sortes. Il y avait les sessions du Comité Technique en mars, juin et novembre et les congrès biennaux, appelés Journées de l'Hydraulique.

Dans les années cinquante, les sessions du Comité Technique étaient occupées par des présentations de communication des membres du Comité Technique ou des représentants de sociétés adhérentes à la SHF. C'était pour beaucoup de jeunes chercheurs un banc d'essai pour présenter leurs travaux, leurs espoirs, leurs idées... et même leurs résultats bien sûr. L'aréopage de chercheurs chevronnés dans l'auditoire était impressionnant pour les orateurs mais les discussions se passaient dans un tel climat de confiance et d'indulgence (sans mettre en risque l'objectivité) que le profit était très fort pour les orateurs et les auditeurs. La participation à ces sessions était gratuite; elle l'est restée jusqu'aux années 80 grâce au mécénat très efficace d'Electricité de France qui mettait gratuitement à disposition des salles et le matériel nécessaire. On se souvient de la petite salle souterraine de la place des EtatsUnis qui vite bondée donnait une impression réconfortante de succès. Il y avait alors à chaque session du Comité Technique une dame sténographe qui prenait mot à mot (ou presque) l'ensemble des discussions revues ensuite par les intervenants avant impression à la suite des exposés. Cela donnait un 
caractère très vivant aux comptes rendus qui paraissaient d'abord dans la revue de la SHF, "Mémoires et Travaux » jusqu'en 1965 puis par la suite dans la Houille Blanche.

C'était la grande époque des publications sur l'eau en français qui était encore dans les années soixante la langue utilisée à égalité avec l'anglais dans les congrès internationaux hors de France (un décompte a été fait par l'un des auteurs qui participait - pour la première fois - à un congrès de la prestigieuse Association Internationale de Recherche Hydraulique dans le site tout aussi prestigieux et encore parfaitement indemne de Dubrovnik à l'été 1961).

Petit à petit le caractère spontané (ou presque) des sessions du Comité Technique s'étiola et les sessions à thème devinrent majoritaires puis exclusives. Mais elles fonctionnaient encore sur appel à communication. Les propositions étaient nombreuses car la séduction et la domination des communications en anglais dans les CV n'avaient pas encore fait les ravages qui ont conduit à l'hégémonie de l'anglais dans les publications scientifiques.

L'autre occasion de réunion proposée par la SHF étaient les Journées de l'Hydraulique toujours à thème mais celui-ci était suffisamment large pour accueillir les travaux d'une forte minorité d'ingénieurs et chercheurs et susciter l'intérêt culturel d'un très nombreux auditoire formé des familiers de la SHF et des nouveaux accrochés par les sujets. Il y avait encore beaucoup d'hydraulique naturelle ou expérimentale dans les communications mais petit à petit l'informatique faisait sa percée. Cénacle fermé d'une poignée d'hydrauliciens aventureux à la fin des années cinquante, outil efficace des jeunes thésards à la fin des années soixante, l'informatique commence à devenir plus populaire dans les années soixante dix avec l'apparition des petites machines individuelles encore balbutiantes à petites cartes magnétiques chez Olivetti, à ruban perforé bien encombrant et fastidieux à modifier puis à cassettes audio chez Wang, elle devient complètement banalisée à la portée de tout le monde dans le début des années quatre-vingt avec l'apparition des premiers Mac Intosh. Et maintenant, les auteurs dans leurs communications ne parlent même plus de l'outil qui leur a permis de montrer de superbes images de la réalité virtuelle hydraulique. Implicitement l'ordinateur est partout, dans les roues des turbines, souterrain dans les nappes, menaçant dans les crues, clapotant avec la pluie statistique mais depuis longtemps il a été affiché explicitement par la SHF. Par exemple dès la session des 8 et 9 juin 1967 avec le thème « Modèles mathématiques d'écoulements fluviaux et maritimes à surface libre " et peu après les 19 et 20 novembre 1970 sous le titre "Modèles mathématiques en hydrologie de surface ». Mais dans cette décennie déjà lointaine l'ordinateur passait encore par le truchement des modèles mathématiques. Ceux-ci sont encore l'intermédiaire dans la session des 15 et 46 novembre 1989 intitulée « Les modèles mathématiques pour la gestion de la qualité des eaux superficielles". Mais l'ordinateur s'affirme ouvertement dans les Journées de l'Hydraulique des 11,12 et 13 Septembre 2000 sous les thèmes « Les logiciels de la mécanique des fluides ».

Pris sous l'angle de vue de l'informatique, la lecture des thèmes des sessions du Comité Technique ou des Journées de l'Hydraulique est anecdotique mais la liste complète des thèmes recensés depuis 1946 bien que cahotante dans les archives d'après guerre est fort intéressante pour démontrer la vitalité et la diversité de la SHF. Pour mieux les faire apprécier de manière synthétique, nous avons essayé de ranger ces thèmes suivant trois grandes classes d'aspect de l'hydraulique: l'hydraulique de l'effort, l'hydraulique du transport, l'hydraulique du confort. Bien sûr comme tout classement, il y a dans nos choix une large part d'arbitraire et de subjectivité. De plus, le classement dépend d'archives parfois lacunaires mais nous avons repéré dans une liste un peu désordonnée une session 057 du Comité Technique des 17 et 18 novembre 1955 sur le thème de la Rhéologie dont les exposés ont été publiés dans un supplément de la revue «Mémoires et Travaux » numéro II 1956. (Cette publication "Mémoires et Travaux » a été la première expression spécifique écrite de la SHF puis a coexisté avec sa sœur « La Houille Blanche " née un peu avant elle en 1902, aujourd'hui centenaire guillerette). Cela signifie donc que depuis sa création en 1912, malgré les aléas catastrophiques de deux guerres mondiales, contre vents et marées la SHF avait tenu 57 sessions en 43 ans ou même en seulement 34 ans si on soustrait les années de guerre, soit presque un rythme de deux réunions scientifiques et techniques par an.

Mais revenons à notre travail d'entomologiste de réunions label SHF.

L'hydraulique de l'effort c'est celle des machines et de la production de l'électricité, fleuron de la SHF dès sa naissance. L'hydraulique de l'effort, c'est en somme la maîtrise de la puissance de l'eau, forme détournée de l'énergie solaire.

L'hydraulique du transport est duale : il y a bien sûr le transport de l'eau et de ce qu'elle contient dans les canalisations en tube, par canaux ou simplement par cours d'eau naturel. Et dans ce dernier cas le transport solide est un thème récurrent important depuis l'érosion des champs amont jusqu'à l'engraissement (ou l'abrasion) des plages. Mais il y a aussi les bateaux graciles ou les lourds convois de péniches.

L'hydraulique du confort c'est celle de l'hygiène et de la santé allant du litre ou la marie-jeanne d'eau quotidiens pour assurer le drainage interne du corps (qui contient environ $75 \%$ d'eau) à l'eau assurant l'assainissement. Hydraulique du confort qui glisse au plaisir avec les piscines, les parcs d'attraction aquatique, les canons à neige et les jets d'eau murmurant sous les arbres ou émigrés au milieu du carrousel urbain des automobiles. Cette hydraulique du confort c'est aussi celle de la protection contre les événements néfastes, crues ou sécheresses, dont celle de la prévision qui va jusqu'au pronostic météorologique.

Suivant cette trilogie, nous avons tenté de suivre par le graphique l'évolution sur la deuxième partie du $\mathrm{XX}^{\mathrm{e}}$ siècle présentée sur la planche. Bien sûr, bien des sessions présentent des thèmes hybrides mais nous avons choisi de ne les rattacher qu'à un seul thème. La terminologie choisie, effort, transport, confort, clin d'œil à la rime, est un peu en porte à faux dans certains cas. L'hydraulique souterraine a été par exemple rattachée au transport. On aurait pu la rapprocher du confort ou plutôt de l'inconfort, vue à travers la migration et la persistance des pesticides.

$\mathrm{Si}$ le lecteur a un peu d'indulgence vis-à-vis de notre traitement flou de l'information, il aura tout de même la preuve des services rendus par le SHF à la communauté scientifique et technique qui s'occupe des problèmes liés à l'eau.

Un chiffre global d'abord: 98 manifestations en 40 ans ! Soit environ 2,5 réunion par an (comme on parle de 1,8 enfants par femme avec l'ISF — indice synthétique de fécondité cher à l'ONU !). 
La planche de synthèse construite à partir de 1960 montre que sur 40 ans le domaine initial de la SHF, l'énergie reste globalement majoritaire. Mais l'observation plus attentive des deux dernières décennies fait apparaître une montée en puissance de la prise en compte des problèmes de société qui ont décollé brusquement dans les années soixante-dix, peut-être comme un contrecoup avec léger retard de la «révolution culturelle " de 1968 (qui avait provoqué d'abord l'annulation de la session de juin du Comité Technique, puis son simple report d'un mois, l'agitation juvénile de la rue passée).

Ce phénomène de la considération des facteurs humains n'est évidemment pas propre à la SHF. C'est un mouvement général. La technique pure et dure est controversée et boudée par les jeunes générations. Elle continue à être un facteur essentiel de progrès matériel par les innovations mais le citoyen de plus en plus individualiste veut qu'elle lui assure aussi la qualité de vie immatérielle.

L'itinéraire historique qui précède est un peu cahotant mais ces cahots sont un peu le reflet de la vie car comme le dit l'un de nous (M. P.) «la SHF a traversé deux guerres et trois républiques " mais en terminant donnons les impressions très subjectives d'un auteur qui fréquenta la SHF assidûment depuis 1959 où il fit rue de Grenelle son premier exposé sur le calcul électronique des intumescences effectué sur l'ordinateur IBM 650 nouvellement installé à la faculté des sciences de Toulouse.

Pour lui, jeune chercheur, la SHF fut le lien privilégié de rencontre entre universitaires, industriels et responsables administratifs s'occupant des problèmes de l'eau. Il a été séduit par la cordialité de l'accueil des jeunes par les caciques de la SHF de l'époque. La SHF a été et doit rester un lieu privilégié d'échanges scientifiques et culturels entre générations mêlant l'humour et l'indulgence. Evidemment l'accélération du progrès informatique fait que la transmission d'expérience se fait moins aisément à trente ou quarante ans de distance mais la culture pragmatique des anciens suggère que la science et la technique en hydraulique ne sont pas faites seulement de la réalité virtuelle de la modélisation mais d'abord d'une observation d'entomologiste depuis la rive ou le pont, des tourbillons et des ressauts.

La SHF c'est l'émulation dans une atmosphère cordiale, la critique vigilante dans la tolérance prospective dans les sessions quasi trimestrielles et surtout les Journées de l'Hydraulique biennales, grandes kermesses scientifiques de l'eau avec très souvent une participation internationale notoire. La SHF avec son écho la Houille Blanche ont été et restent l'ambassadeur de la France dans le domaine de l'eau. Bien sûr, la prolifération des associations nationales ou internationales et la suprématie envahissante de la langue anglaise ont un peu émoussé le rayonnement éclatant de la SHF mais celle-ci a su créer des liens et symbioses avec les «start up » associatives telles le conglomérat Association de Mécanique des Fluides, la vénérable Société des Thermiciens, ou le lilliputien GFHN.

Souvent futuriste, toujours pragmatique, la SHF d'aujourd'hui a continué dans l'esprit de l'entre deux guerres à servir de bureau d'étude et de laboratoire aux Ministères (par exemple pour les crues) ou aux constructeurs, par exemple pour la concertation d'essais et la définition de normes pour les machines hydrauliques (par exemple dans leur fonctionnement à charge partielle).

La SHF : des faits, des hommes, des idées. Une histoire à continuer à écrire pour et par les générations du $\mathrm{XXI}^{\mathrm{e}}$ siècle ! 